\title{
La gestión del conocimiento y los sistemas de información como fuentes de ventaja competitiva para las empresas
}

\section{Knowledge management and information systems as sources of competitive advantage for companies}

María Auxiliadora Guerrero Bejarano, MBA

Universidad Internacional del Ecuador, Ecuador

Daniel Ricardo Silva Siu, Msc

Universidad ESAN, Perú

Autor por Correspondencia: dsilva@pobox.com, maguerrerobe@uide.edu.ec

Fecha de recepción: 31 de Enero de 2017 - Fecha de aceptación: 25 de Marzo de 2017

\section{Resumen}

Esta investigación teórica busca resaltar la importancia de la gestión del conocimiento y los sistemas de información en las empresas y cómo a partir de ellas, las organizaciones pueden obtener ventajas competitivas que les permitan mantenerse y desarrollarse en el mercado.

Palabras claves: gestión del conocimiento; ventajas competitivas; desarrollo de mercado

\begin{abstract}
This theoretical research seeks to highlight the importance of knowledge management and information systems in companies and how from them, organizations can gain competitive advantages that allow them to stay and develop in the market.
\end{abstract}

Key words: knowledge management; competitive advantages; market development 


\section{Introducción}

En el mercado actual, donde la globalización juega un papel cada vez más importante en el desarrollo de las industrias, las organizaciones se enfrentan a cambios que generalmente son el efecto de factores que los empresarios no pueden controlar, muchos de estos cambios se deben a la evolución tecnológica que influye en la forma en la que se hacen las cosas. Las empresas van evolucionando debido a estos cambios y generando nuevos procesos sobre la base de los conocimientos que adquieren en su aprendizaje; este análisis teórico busca resaltar cómo el manejo de esta información, de estos conocimientos adquiridos, pueden ser una fuente de ventaja competitiva para las organizaciones. El conocimiento sobre los clientes y sobre cómo hacer las cosas resultarían claves en el desarrollo de las empresas.

La gestión del conocimiento es la forma en la que se identifica y aprovecha el saber en una empresa para facilitar la competitividad de la misma, existen dos tipos de conocimiento, el tácito y el implícito; las empresas están llamada a definir estrategias para manejar cada tipo de conocimiento de acuerdo con las características de cada negocio (Bejarano, 2016)

Se identifican cuatro factores que de forma dinámica interactúan en la gestión del conocimiento, que permite obtener el crecimiento de la organización: (a) de tácito a tácito, este se da cuando los individuos mediante la observación, imitación y/o práctica, comparten conocimiento; no es sistematizado por lo que no puede ser usado por la empresa como explícito; (b) de explícito a explícito, combina elementos del conocimiento existente, adaptando una nueva perspectiva del todo, no implica la expansión de la base del conocimiento de la organización; (c) de tácito a explícito, se produce cuando un individuo es capaz de articular los fundamentos de su conocimiento tácito y lo convierte en explícito permitiendo el crecimiento de la base del conocimiento de la organización; (d) de explícito a tácito, se da cuando el conocimiento explícito es compartido por toda la organización, al ser utilizado por todos se amplía, extiende y es incorporado en su propio conocimiento tácito (Nonaka, 2008). Las organizaciones deben transformarse en sociedades del conocimiento, entendiendo que este es un nuevo factor de producción, que permite el desarrollo de tecnologías, metodologías y estrategias, convirtiéndose en una prioridad para las empresas, debiendo establecerse formas para gestionarse y medirse (Gómez, 2006; Viedma, 2000).

Algunos autores reconocen la importancia del estilo del liderazgo empleado por los CEOs de las organizaciones en la gestión del conocimiento y el uso de este con los sistemas de información (Kuo, Lai, \& Lee, 2011; Lakshman, 2009); los gerentes deben buscar la manera de empoderar a sus colaboradoras para facilitar el desarrollo del ambiente de la organización dónde éstos puedan participar en las acciones que fomenten la gestión del conocimiento de forma más espontánea (Anantatmula, 2008). Sin este apoyo por parte de los gerentes, a pesar de la existencia de procesos, podrían no obtenerse los resultados deseados (Kuo, 2013), otros reconocen que la gestión del conocimiento influenciada por el liderazgo puede generar empresas más orientadas hacia la innovación (Darroch \& McNaughton, 2002; García-Morales, JiménezBarrionuevo, \& Gutiérrez-Gutiérrez, 2012; González-Sánchez \& García-Muiña, 2011). La aparición y creciente importancia del conocimiento como un nuevo factor de producción hace que el desarrollo de esas tecnologías y metodologías para la medición y difusión han convertido el conocimiento en un elemento indispensable para el desarrollo económico y social (Gómez, 2006). 
La gestión del conocimiento estaría determinada por cinco factores que garantizarían su éxito: (a) cultura organizacional orientada al conocimiento, que identifica tres componentes, la orientación positiva hacia el conocimiento, la ausencia de factores de inhibición del conocimiento en la cultura y que el tipo de proyecto de gestión del conocimiento coincida con la cultura; (b) infraestructura técnica e institucional, que significa poseer tecnología adecuada que permita una implantación más sencilla de la gestión del conocimiento; (c) respaldo del personal directivo, que resulta fundamental para el éxito del KM; (d) vínculo con el valor económico, que significa que estos procesos deben significar beneficios para la organización y (e) orientación del proceso (Gómez, 2006).

El avance tecnológico ha marcado un ritmo acelerado en el que la práctica de las empresas ha ido avanzando, los sistemas de información se ha ido desarrollando en función con la evolución de las empresas y sus necesidades, los primeros períodos en los que se registran actividades relacionadas con el manejo de la información coinciden con la teoría económica y la organización industrial, cuyos supuestos establecen que las organizaciones compiten y por esto buscan obtener mejores resultados (Porter \& Kramer, 2006). Un mejor conocimiento de los clientes, saber determinar cómo hacer mejores negocios con ellos, permite a las organizaciones obtener mejores resultados y ganar ventajas competitivas (Lakshman, 2009; Lesser, Mundel, \& Wiecha, 2000).

Con respecto a los sistemas de información, existe una evolución técnica inminente que ha ido cambiando la forma en la que las empresas hacen las cosas, afectando la economía y las finanzas de las organizaciones, con la aparición de aplicaciones más centradas en la gestión de la información y conocimiento que necesariamente en las operaciones y la producción, lo que denota la convergencia entre los sistemas de información y la gestión del conocimiento (Bárcena \& del Campo, 2002; Pérez \& Dressler, 2007; Salazar, 2000).

\section{Conclusiones}

La gestión del conocimiento identifica y aprovecha el conocimiento colectivo generado en una empresa para facilitar el nivel de competitividad de la misma, por este motivo se debe considerar los tipos de conocimiento a los que se hizo referencia al inicio de este trabajo, la organización establecerá entonces las diferentes estrategias con las que gestionará el conocimiento de acuerdo con el modelo de negocio, si son empresas orientadas al servicio, así como si elaboran productos, el conocimiento de sus clientes se convertirá en un elemento se su cadena de valor; por lo que se podría afirmar que las estrategias para cada tipo de conocimiento no serían excluyentes, por el contrario, sino complementarias y facilitan la adecuada gestión de ambos tipos de conocimiento. Existirían claras evidencias en los distintos estudios que demuestran la importancia de la gestión del conocimiento en el éxito de las organizaciones, medidos por la posibilidad de obtener ventajas frente a los competidores, dadas por la calidad del servicio, habilidades técnicas y la capacidad del recursos hacia la adaptabilidad y flexibilidad, influenciados por el estilo del liderazgo y los valores aplicados en la empresa, que determinan los procesos y sistemas de información que se emplean para gestionar el conocimiento que se produce en la organización (Darroch \& McNaughton, 2002; González-Sánchez \& García-Muiña, 2011; Haldma, Näsi, Grossi, Saunila, \& Ukko, 2012; Salazar, 2000; Simón, Kumar, Schoeman, Moffat, \& Power, 2011) 


\section{Bibliografía}

Anantatmula, V. S. (2008). Leadership role in making effective use of km. VINE, 38(4), 445-460.

Bárcena, L. S., \& del Campo, M. A. M. (2002). La estructura de incentivos y los mecanismos de coordinación para una gestión eficiente del conocimiento. Boletín de Estudios Económicos, 57(176), 227.

Bejarano, M. A. G. (2016). La gestión del conocimiento en las empresas, su importancia y dependencia del estilo del liderazgo de la alta gerencia. INNOVA Research Journal, 1(1).

Darroch, J., \& McNaughton, R. (2002). Examining the link between knowledge management practices and types of innovation. Journal of intellectual capital, 3(3), 210-222.

Garcia-Morales, V. J., Jiménez-Barrio Nuevo, M. M., \& Gutiérrez-Gutiérrez, L. (2012). Transformational leadership influence on organizational performance through organizational learning and innovation. Journal of business research, 65(7), 1040-1050.

Gómez, D. R. (2006). Modelos para la creación y gestión del conocimiento: Una aproximación teórica.

González-Sánchez, R., \& García-Muiña, F. E. (2011). Innovación abierta: Un modelo preliminar desde la gestión del conocimiento. Intangible capital, 7(1), 82-115.

Haldma, T., Näsi, S., Grossi, G., Saunila, M., \& Ukko, J. (2012). A conceptual framework for the measurement of innovation capability and its effects. Baltic Journal of Management, 7(4), 355-375.

Kuo, R.-Z., Lai, M.-F., \& Lee, G.-G. (2011). the impact of empowering leadership for kms adoption. Management Decision, 49(7), 1120-1140.

Kuo, T.-H. (2013). How expected benefit and trust influence knowledge sharing. Industrial Management \& Data Systems, 113(4), 506-522.

Lakshman, C. (2009). Organizational knowledge leadership: An empirical examination of knowledge management by top executive leaders. Leadership \& Organization Development Journal, 30(4), 338364.

Lesser, E., Mundel, D., \& Wiecha, C. (2000). Managing customer knowledge. Journal of Business Strategy, 21(6), 34-37.

Nonaka, I. (2008). The knowledge-creating company: Harvard Business Review Press.

Pérez, D., \& Dressler, M. (2007). Tecnologías de la información para la gestión del conocimiento. Pérez, Daniel; Dressler, Matthias." Tecnologías de la información para la gestión del conocimiento". Intangible Capital, enero-marzo de 2007, vol. 3, núm. 15, p. 31-59.

Porter, M. E., \& Kramer, M. R. (2006). Estrategia y sociedad. Harvard Business Review, 84(12), 42-56.

Simon, A., Kumar, V., Schoeman, P., Moffat, P., \& Power, D. (2011). Strategic capabilities and their relationship to organizational success and its measures: Some pointers from five Australian studies. Management Decisión, 49(8), 1305-1326.

Viedma, J. M. (2000). La gestión del conocimiento y del capital intelectual. Nueva Empresa. Com N454, 22, 99-105. 